\title{
Isotope Shifts and Hyperfine Structure of Erbium, Dysprosium, and Gadolinium by Atomic-Beam Diode-Laser Spectroscopy
}

\author{
R. J. Lipert, S. C. Lee \\ Appl. Phys. B 57, 373-379 (1993)
}

Due to a compositor's error, on page 374 of this paper

\section{Discussion}

For rare-earth elements, the isotope shift in a spectral line can be caused by both mass dependent effects and volume or field effects. The mass dependent effects are further divided into contributions from the Normal Mass Shift (NMS) and Specific Mass Shift (SMS). For both erbium and dysprosium, the spectral lines studied here involved $4 f-5 d$ transitions [25]. This type of transition normally has a relatively large SMS [28].

was printed. It should read:

\section{Results}

The transitions studied in the current investigation are summarized in Table 1 and the spectra obtained for erbium and dysprosium are shown in Figs. 1 and 2, respectively. For the gadolinium transition at $773.35 \mathrm{~nm}$, the congestion was so severe that no resolvable hyperfine structure was apparent. The most prominent features in the gadolinium spectrum are transitions due to the three most abundant, even mass number isotopes. Even these lines are probably overlapped with some hyperfine transitions. 